\title{
Percutaneous Endoscopic Transforaminal Discectomy with Foraminoplasty in the Treatment of L4-L5 and L5-S1 Disc Herniation: A Minimum 1-year Follow-up Study.
}

\section{Haowei Jiang}

Zhejiang University Huzhou Hospital: Huzhou Central Hospital

\section{Yongli Wang}

Zhejiang University Huzhou Hospital: Huzhou Central Hospital

\section{Tianshun Fang}

Zhejiang University Huzhou Hospital: Huzhou Central Hospital Jiangbo Nie

Zhejiang University Huzhou Hospital: Huzhou Central Hospital

Mingchao Jin

Zhejiang University Huzhou Hospital: Huzhou Central Hospital

Qian Lu

Zhejiang University Huzhou Hospital: Huzhou Central Hospital

Xuesheng Jiang (D253555625@qq.com )

Huzhou central hospital

Research article

Keywords: Lumbar disc herniation, Percutaneous Endoscopic Transforaminal Discectomy with Foraminoplasty, clinical outcomes, radiographic measurements

Posted Date: May 10th, 2021

DOI: https://doi.org/10.21203/rs.3.rs-459110/v1

License: (c) (i) This work is licensed under a Creative Commons Attribution 4.0 International License. Read Full License 


\section{Abstract \\ Background}

The anatomic and biomechanical aspects of the L5-S1 level present unique operative challenges compared with the L4-L5 level. We aim to explore the clinical outcomes and radiographic measurements of L4-L5 patients compared with L5-S1 patients after undergoing Percutaneous Endoscopic Transforaminal Discectomy with Foraminoplasty (PETDF).

\section{Methods}

A total of 84 patients who underwent PETDF for LDH treatment between January 2017 and June 2020 were included in this study. Preoperative, perioperative, demographic data, clinical and radiographic outcomes were compared between patients with L4-L5 involvement and patients with L5-S1 involvement.

\section{Results}

There were no significant differences between the two groups in terms of Age, Gender, Follow-up time, the postoperative questionnaire results (Visual Analog Scale [leg], VAS [lower back], and Oswestry Disability Index) or the Excellence and Good Rate and Recurrence Rate. There was no significant difference in the mean preoperative to postoperative change in Intervertebral Space Height (ISH), Intervertebral Space Angle (ISA), Lumbar Lordosis, Foraminal Area (FA) of Operating Side and FA of Contralateral Side between the 2 groups. The mean VAS of Leg Pain, VAS of Lower Back Pain and ODI postoperative scores were significantly improved over the preoperative scores in each of the two groups and the ISH, ISA, FA of Operating Side and FA of Contralateral Side postoperative were significant difference form preoperative

\section{Conclusion}

PETDF is an effective and safe treatment approach for lumbar disc herniation in both L4/L5 and L5/S1 level. Although it may increase lumbar ISA and result in low CFA (contralateral foraminal area) and ISH.

\section{Background}

Lumbar disc herniation (LDH), a common degenerative spinal disease ${ }^{1}$, is characterized by displacement of intervertebral disk material beyond the normal margins of the disk space. Approximately $95 \%$ of reported LDH cases are L4-L5 or L5-S1 levels².

Percutaneous Endoscopic Transforaminal Discectomy (PETD) is the conventional treatment approach for lumbar disc herniation. PETD is more effective compared with traditional open lumbar discectomy. Its advantages include less paravertebral muscle injury, preservation of bony structures, and rapid recovery. 
Foraminoplasty spinal surgery expands the neuro foramen ensuring convenient and effective operation of herniation ${ }^{3}$. Extensive foraminoplasty is required for patients who suffer from hypertrophy of the facet joints, for decompression of exiting nerve roots ${ }^{4}$. However, foraminoplasty may result in injury of the facet joint, generating postoperative lower back pain, and increasing lumbar instability and high recurrence rate post-operation ${ }^{4-7}$.

Previous studies report conflicting findings on clinical outcomes and complications for L4-L5 patients undergoing full-endoscopic lumbar discectomy compared with patients with L5-S1 level ${ }^{8,9}$. Biomechanical studies on discectomy procedures of lumbar spine report differences in load resistances at each lumbar segment ${ }^{10}$. However, studies have not explored the clinical outcomes and radiographic measurements of L4-L5 patients compared with L5-S1 patients after undergoing Percutaneous Endoscopic Transforaminal Discectomy with Foraminoplasty (PETDF) surgery. Therefore, we carried out a retrospective study, comprising 84 patients who underwent PETDF and compared differences in clinical outcomes and radiographic measurements between L4-L5 group and L5-S1 group after at least one-year follow-up.

\section{Methods}

\subsection{Patients}

This study was approved by our institutional review board. A total of 84 patients who underwent PETDF for LDH treatment between January 2017 and June 2020 were included in this study. The inclusion criteria were: (1) single-level (L4-L5 or L5-S1) lumbar disc herniation, (2) radiating pain in the unilateral lower limb, (3) Magnetic Resonance Imaging (MRI) indicating herniated disc at L4-L5 or L5-S1, in agreement with clinical symptoms and signs and (4) failure of $\geq 6$ weeks of conservative treatment. Exclusion criteria included: (1) spinal deformity or spinal instability and (2) recurrent LDHs

\section{2 surgery}

All surgeries were performed by one senior and experienced surgeon. Patients were placed in a lateral position on a radiolucent table in the operating suite with the affected side facing upwards. All surgeries were performed under local anesthesia. Direction and site of puncture were marked under fluoroscopic guidance. An 18-gauge spinal needle was inserted into the target disc level under fluoroscopic guidance. The needle tip was placed at the posterior edge of the target disc space on the lateral view whereas the needle tip was placed at the medial pedicular line on the anterio-posterior view. A guidewire was inserted into the spinal needle then the spinal needle was removed. A dilating obturator was passed over the guidewire. A $7.5 \mathrm{~mm}$ trephine was then used to cut off the anterior part of the superior articular process to facilitate insertion of the working cannula. Further, an endoscope was introduced through the cannula. The herniated disc was resected using endoscopic forceps and a bipolar radiofrequency coagulator.

\subsection{Outcome measurements}


Radiographs were assessed preoperatively and at the final follow-up. Radiologic study parameters included intervertebral space height (ISH) (Figure.1), intervertebral space angle (ISA), foraminal area (FA) (The Foraminal Area was measured on T2-weighted MRI at the most stenotic sagittal plane) and lumbar lordosis.

MacNab criteria were used to evaluate surgical effectiveness. Visual analog scale (VAS) and Oswestry disability index (ODI) were used to assess low-back pain and leg pain preoperatively and postoperatively during regular follow-ups at 1 week, 1 month and at least 1 year.

\section{Results}

\subsection{Demographic characteristics of patients}

A total of 84 patients (L4-L5 group, 40 patients; L5-S1 group, 44 patients) were enrolled in this study. Preoperative demographics were not significantly different $(P>0.05)$ between the two groups (Table 1$)$.

\subsection{Clinical outcomes}

The mean follow-up period was $13.46 \pm 1.94$ months (range: $12-21$ months). Postoperative pain scores using VAS and ODI showed a significant improvement in leg and lower back pain in both groups compared with preoperative scores $(P<0.05$, Table 2 and Table 3). Preoperative and Postoperative VAS and ODI scores were not significantly different for the two groups $(P>0.05$, Table 1$)$.

The 'excellent' and 'good' rates based on Macnab criteria were not significant different for L4-L5 group (85.00\%) compared with the L5-S1 group $(90.91 \%)$ at the last follow-up $(\chi 2=0.70, P=0.40$, Table 1$)$.

The recurrence rate was not significantly different for the L4-L5 group (2.50\%) compared with the recurrence rate for the L5-S1 group (4.55\%) at the last follow-up ( $\chi 2=0.26, P=0.61$, Table 1$)$.

\subsection{Radiologic outcomes}

\subsubsection{Intervertebral space height (ISH) and Intervertebral space angle (ISA)}

ISH decreased significantly in two groups $(P<0.05$, Table 2 and Table 3$)$.

ISA increased significantly in two groups $(P<0.05$, Table 2 and Table 3$)$

The mean preoperative to postoperative change in ISH and ISA were not significant different $(P>0.05$, Table 1) for the two groups

\subsubsection{Lumbar lordosis}

Preoperative and postoperative lumbar lordosis were not significant different $(P>0.05$, Table 1$)$ for the two groups. No significant improvement on lumbar lordosis was observed for both groups $(P>0.05$, 
Table 2 and Table 3).

\subsubsection{Foraminal area (FA)}

Preoperative and postoperative FA were not significant different $(P>0.05$, Table 1$)$ for the two groups

FA of the operating side increased significantly in all patients after PETDF $(P<0.05$, Table 2 and Table 3$)$.

FA of the contralateral side decreased significantly in all patients after PETDF $(P<0.05$, Table 2 and Table 3).

\section{Discussion}

Percutaneous Endoscopic Transforaminal Discectomy (PETD) was developed after Yeung et al ${ }^{11}$ and Hoogland et $\mathrm{al}^{3}$ introduced the Yeung endoscopic spine system (YESS) and the transforaminal endoscopic spine system (TESSYS), respectively. Foraminoplasty techniques are effective surgical approaches for improving indications of PETD ${ }^{5}$. Knight, Martin T. N. et al ${ }^{12}$ report that PETD without foraminoplasty aggravates incipient lateral recess or foraminal stenosis. PETDF is associated with good clinical outcomes ${ }^{12,13}$. However, $\mathrm{Li} \mathrm{J}$ et al ${ }^{4}$ report that all biomechanical indexes are deteriorated in the three-dimensional lumbosacral model with one-half excision of the superior articular process. These findings indicate that foraminoplasty may increase risk of biomechanical deterioration. Qiao P et al ${ }^{5}$ report that PETDF in L4-L5 increases lumbar instability and postoperative recurrence rates. Currently, at least $95 \%$ of LDH cases occur at the L4-L5 or L5-S1 segment. Therefore, the differences in clinical outcomes and radiographic measurements due to different levels of disc herniation in patients after undergoing PETDF should be explored.

The clinical outcomes of PETDF recorded in this study were similar to findings reported in previous studies $^{12-14}$. Analysis showed significant improvements in pain score and functional status of all patients during the final follow-up. These findings imply that PETDF is effective in relieving both low back and leg pain in patients with LDH.

Interestingly, contralateral foraminal area (CFA) significantly decreased in all patients at the final followup. A few studies have explored degeneration of contralateral intervertebral foramen and lateral recess after PELDF. Lumbar facet joints are the conventional methods for guiding and stabilizing the spinal motion segment, especially in maintaining lumbar stability in forward flexion ${ }^{15-17}$. A previous study using nucleotomy model ${ }^{18}$ reports that facet joints supported significantly greater load after nucleotomy. Supported load (15.8\%) was almost doble the applied external force (8.6\% of). Notably, low quantity of nucleus removal (range: $0.7-1.7 \mathrm{~g}$ ) increased the forces transmitted over the facet joints. Previous studies report that destruction of lumbar facet joints results in increase in rotation, loss of strength, and decrease in stiffness of spinal motion segment ${ }^{16,19,20}$. Moreover, facetectomy results in facet joint diseases and lower back pain, especially on the contralateral side 4 . Therefore, discectomy and foraminoplasty performed during PETDF may result in increased contralateral facet joint loading in order to maintain 
spinal mechanical stability in a variety of directions and loading scenarios. This increase in contralateral facet joint loading ultimately results in decreased CFA.

Maintenance of Intervertebral space height (ISH) is a good prognostic index after PETD ${ }^{21,22}$. However, several studies on PETD report reduction of ISH post-operation ${ }^{5,23}$. Reduction of ISH is attributed to loss of the nucleus-pulposus volume; loss of nucleus-pulposus constituent due to use of bipolar radiofrequency, including loss of mucopolysaccharide protein complex, chondroitin sulfate and high amounts of water; defects in annulus fibrosus during surgical discectomy; and intervertebral disc degeneration ${ }^{5}$. In our study, ISH decreased in the two groups. Eun, Sang Soo et al ${ }^{23}$ report that the average postoperative disc-height ratio in patients (73.7\% patients were at L4-L5 spinal level, 10.5\% patients were at L5-S1 spinal level) who underwent PELD was $81.54 \%$ of the original disc height at 10 years prospective follow-up. These findings were consistent to findings of our study. In addition, Qiao $\mathrm{P}^{5}$ reports reduction of ISH post-operation in 64 patients with L4/5 spinal level who underwent PETDF. These findings show that higher reductions of ISH are correlated with higher increase in intervertebral space angle (ISA). Furthermore, pain is correlated with decrease in lumbar ISA ${ }^{24}$, whereas ISA increases with decrease in pain after operation. ${ }^{5}$ Although, ISA increased in the two groups, ISA was not correlated with decrease in ISH. This finding can be attributed to the small sample size.

This study has several limitations. First, there may be errors associated with measuring the FA on MRI. We measured the FA in the sagittal T2 images at the most stenotic sagittal plane. However, these sagittal images may be heterogeneous due to differences in the cutting angle of the MRI resulting from individual anatomic variations and technical problems. A $3.0 \mathrm{~mm}$ slice of sagittal T2-weighted MR image is also thicker than an ideal slice. Second, this study only used FA measurement. Therefore, our results may be limited regarding measurement of the nerve root pressure or morphologic changes. Finally, another limitation of this study is its retrospective nature.

\section{Conclusion}

PETDF is an effective and safe treatment approach for lumbar disc herniation in both L4/L5 and L5/S1 level. However, it may increase lumbar ISA (intervertebral space angle) and result in low CFA (contralateral foraminal area) and ISH (intervertebral space height). L4-L5 and L5-S1 level patients showed no difference in clinical outcomes and radiographic measurements despite biomechanical and anatomic differences between these levels.

\section{Abbreviations}

LDH: Lumbar disc herniation; PETD: Percutaneous Endoscopic Transforaminal Discectomy; PETDF: Percutaneous Endoscopic Transforaminal Discectomy with Foraminoplasty; MRI: Magnetic Resonance Imaging; ISH: intervertebral space height; ISA: intervertebral space angle; FA: foraminal area; VAS: Visual analog scale; ODI: Oswestry disability index; CFA: contralateral foraminal area. 


\section{Declarations}

\section{Acknowledgements}

We gratefully acknowledge the support of all doctors in our department.

\section{Availability of data and materials}

The datasets used and analysed during the current study are available from the corresponding author on reasonable request.

\section{Ethics approval and consent to participate}

The study was approved by the Institutional Review Board of Huzhou Central Hospital. The requirement for informed consent was waived due to the retrospective design of our study.

\section{Consent for publication}

Not applicable.

\section{Competing interests}

There are no conflicts of interest to declare.

\section{Funding}

This work is supported by grant from Public Welfare Applied Research Project of Huzhou Science and Technology Bureau (Grant No. 2019GY16).

\section{References}

1. DS K, SW H, JE E, et al. An evidence-based clinical guideline for the diagnosis and treatment of lumbar disc herniation with radiculopathy. The spine journal : official journal of the North American Spine Society. 2014;14(1):180-191.

2. RA D, SK M. CLINICAL PRACTICE. Herniated Lumbar Intervertebral Disk. The New England journal of medicine. 2016;374(18):1763-1772.

3. Hoogland T, Schubert M, Miklitz B, Ramirez A. Transforaminal posterolateral endoscopic discectomy with or without the combination of a low-dose chymopapain: a prospective randomized study in 280 consecutive cases. Spine. 2006;31(24):E890-E897.

4. Li J, Zhang X, Xu W, et al. Reducing the extent of facetectomy may decrease morbidity in failed back surgery syndrome. BMC musculoskeletal disorders. 2019;20(1):369.

5. Qiao P, Xu T, Zhang W, et al. Foraminoplasty affects the clinical outcomes of discectomy during percutaneous transforaminal endoscopy: a two-year follow-up retrospective study on 64 patients. 
The International journal of neuroscience. 2020:1-6.

6. $\mathrm{Li} \mathrm{J,} \mathrm{Li} \mathrm{H,} \mathrm{He} \mathrm{Y,} \mathrm{et} \mathrm{al.} \mathrm{The} \mathrm{protection} \mathrm{of} \mathrm{superior} \mathrm{articular} \mathrm{process} \mathrm{in} \mathrm{percutaneous} \mathrm{transforaminal}$ endoscopic discectomy should decreases the risk of adjacent segment diseases biomechanically. 2020;79:54-59.

7. Zhao Y, Yuan S, Tian Y, Liu XJBjon. Necessity of routinely performing foraminoplasty during percutaneous endoscopic transforaminal discectomy (PETD) for lumbar disc herniation. 2020:1-7.

8. Hua W, Tu J, Li S, et al. Full-endoscopic discectomy via the interlaminar approach for disc herniation at L4-L5 and L5-S1: An observational study. Medicine. 2018;97(17):e0585.

9. Hua W, Zhang Y, Wu X, et al. Full-Endoscopic Visualized Foraminoplasty and Discectomy Under General Anesthesia in the Treatment of L4-L5 and L5-S1 Disc Herniation. Spine. 2019;44(16):E984E991.

10. Okoro T, Sell P. A short report comparing outcomes between L4/L5 and L5/S1 single-level discectomy surgery. J Spinal Disord Tech. 2010;23(1):40-42.

11. Yeung AT, Tsou PM. Posterolateral endoscopic excision for lumbar disc herniation: Surgical technique, outcome, and complications in 307 consecutive cases. Spine. 2002;27(7):722-731.

12. Knight MTN, Jago I, Norris C, Midwinter L, Boynes C. Transforaminal endoscopic lumbar decompression \& foraminoplasty: a 10 year prospective survivability outcome study of the treatment of foraminal stenosis and failed back surgery. International journal of spine surgery. 2014;8.

13. Ahn Y, Oh H-K, Kim H, Lee S-H, Lee H-N. Percutaneous endoscopic lumbar foraminotomy: an advanced surgical technique and clinical outcomes. Neurosurgery. 2014;75(2).

14. Chen P, Hu Y, Li Z. Percutaneous endoscopic transforaminal discectomy precedes interlaminar discectomy in the efficacy and safety for lumbar disc herniation. Biosci Rep. 2019;39(2).

15. Jaumard NV, Welch WC, Winkelstein BA. Spinal facet joint biomechanics and mechanotransduction in normal, injury and degenerative conditions. J Biomech Eng. 2011;133(7):071010.

16. Serhan HA, Varnavas G, Dooris AP, Patwadhan A, Tzermiadianos M. Biomechanics of the posterior lumbar articulating elements. Neurosurg Focus. 2007;22(1):E1.

17. Haher TR, O'Brien M, Dryer JW, Nucci R, Zipnick R, Leone DJ. The role of the lumbar facet joints in spinal stability. Identification of alternative paths of loading. Spine. 1994;19(23).

18. Ivicsics MF, Bishop NE, Püschel K, Morlock MM, Huber G. Increase in facet joint loading after nucleotomy in the human lumbar spine. J Biomech. 2014;47(7):1712-1717.

19. Abumi K, Panjabi MM, Kramer KM, Duranceau J, Oxland T, Crisco JJ. Biomechanical evaluation of lumbar spinal stability after graded facetectomies. Spine. 1990;15(11):1142-1147.

20. Tender GC, Kutz S, Baratta R, Voorhies RM. Unilateral progressive alterations in the lumbar spine: a biomechanical study. Journal of neurosurgery Spine. 2005;2(3):298-302.

21. Sharma SB, Lin G-X, Jabri H, et al. Radiographic and clinical outcomes of huge lumbar disc herniations treated by transforaminal endoscopic discectomy. Clinical neurology and neurosurgery. 2019;185:105485. 
22. Choi KC, Kim J-S, Park C-K. Percutaneous Endoscopic Lumbar Discectomy as an Alternative to Open Lumbar Microdiscectomy for Large Lumbar Disc Herniation. Pain physician. 2016;19(2):E291-E300.

23. Eun SS, Lee S-H, Sabal LA. Long-term Follow-up Results of Percutaneous Endoscopic Lumbar Discectomy. Pain physician. 2016;19(8):E1161-E1166.

24. Endo K, Suzuki H, Tanaka H, Kang Y, Yamamoto K. Sagittal spinal alignment in patients with lumbar disc herniation. European spine journal : official publication of the European Spine Society, the European Spinal Deformity Society, and the European Section of the Cervical Spine Research Society. 2010;19(3):435-438.

\section{Tables}

Table. 1 Patient demographics and Comparison of clinical follow-up outcomes between L4-L5 and L5-S1 groups

\begin{tabular}{|lllll|}
\hline & & L4-L5 (n=40) & L5-S1 $(\mathrm{n}=44)$ & P value \\
\hline Age(year) & & $51.93 \pm 15.91$ & $47.94 \pm 13.80$ & 0.29 \\
\hline Gender (M/F) & $21 / 19$ & $20 / 24$ & $0.52\left(\chi^{2}=0.42\right)$ \\
\hline Follow-up time(month) & & 13.38 & 13.53 & 0.76 \\
\hline VAS of Leg Pain & Pre-op & $5.90 \pm 0.98$ & $5.91 \pm 1.03$ & 0.95 \\
\cline { 2 - 5 } & Post-op & $0.83 \pm 1.47$ & $0.38 \pm 0.60$ & 0.14 \\
VAS of Lower Back Pain & Pre-op & $7.10 \pm 1.65$ & $7.18 \pm 1.93$ & 0.87 \\
\cline { 2 - 5 } & Post-op & $1.21 \pm 1.99$ & $0.59 \pm 0.70$ & 0.12 \\
\hline ODI & Pre-op & $62.62 \pm 11.29$ & $64.12 \pm 11.94$ & 0.29 \\
\cline { 2 - 5 } & Post-op & $15.17 \pm 22.05$ & $6.12 \pm 9.37$ & 0.06 \\
\hline Excellence and Good Rate & & $34 / 40(85.00 \%)$ & $40 / 44 \llbracket 90.91 \% 区$ & $0.40\left(\chi^{2}=0.70\right)$ \\
\hline Recurrence Rate & & $1 / 40(2.50 \%)$ & $2 / 44(4.55 \%)$ & $0.61\left(\chi^{2}=0.26\right)$ \\
\hline
\end{tabular}

Table. 2 Comparison of radiologic follow-up outcomes between L4-L5 and L5-S1 groups 


\begin{tabular}{|c|c|c|c|c|}
\hline & & L4-L5 (n=40) & L5-S1(n=44) & $\begin{array}{l}P \\
\text { value }\end{array}$ \\
\hline \multirow[t]{3}{*}{ Intervertebral Space Height (mm) } & Pre-op & $10.27 \pm 1.97$ & $10.70 \pm 1.75$ & 0.36 \\
\hline & Post-op & $9.92 \pm 1.89$ & $10.01 \pm 1.85$ & 0.85 \\
\hline & $\begin{array}{l}\text { Mean } \\
\text { change }\end{array}$ & $0.34 \pm 0.86$ & $0.69 \pm 1.28$ & 0.21 \\
\hline \multirow[t]{3}{*}{ Intervertebral Space Angle $\left(^{\circ}\right)$} & Pre-op & $6.40 \pm 3.08$ & $10.58 \pm 5.20$ & 0.00 \\
\hline & Post-op & $7.64 \pm 3.25$ & $12.37 \pm 4.98$ & 0.00 \\
\hline & $\begin{array}{l}\text { Mean } \\
\text { change }\end{array}$ & $1.24 \pm 2.26$ & $1.79 \pm 4.28$ & 0.52 \\
\hline \multirow[t]{3}{*}{ Lumbar Lordosis $\left(^{\circ}\right)$} & Pre-op & $34.16 \pm 10.50$ & $30.32 \pm 11.05$ & 0.17 \\
\hline & Post-op & $34.34 \pm 10.75$ & $32.30 \pm 9.46$ & 0.43 \\
\hline & $\begin{array}{l}\text { Mean } \\
\text { change }\end{array}$ & $0.18 \pm 6.38$ & $1.98 \pm 6.07$ & 0.26 \\
\hline \multirow{3}{*}{$\begin{array}{l}\text { Foraminal Area of Operating Side } \\
\left(\mathrm{mm}^{2}\right)\end{array}$} & Pre-op & $132.74 \pm 38.59$ & $130.84 \pm 30.12$ & 0.83 \\
\hline & Post-op & $151.50 \pm 46.81$ & $142.77 \pm 37.92$ & 0.42 \\
\hline & $\begin{array}{l}\text { Mean } \\
\text { change }\end{array}$ & $18.76 \pm 46.81$ & $11.93 \pm 30.77$ & 0.49 \\
\hline \multirow[t]{3}{*}{ Foraminal Area of Opposite Side $\left(\mathrm{mm}^{2}\right)$} & Pre-op & $132.96 \pm 34.27$ & $136.94 \pm 37.05$ & 0.66 \\
\hline & Post-op & $123.19 \pm 31.92$ & $125.85 \pm 33.15$ & 0.75 \\
\hline & $\begin{array}{l}\text { Mean } \\
\text { change }\end{array}$ & $-9.77 \pm 21.84$ & $-11.08 \pm 27.88$ & 0.84 \\
\hline
\end{tabular}

Table. 3 Comparison of clinical and radiologic outcomes of L4-L5 groups pre-operative and postoperative 


\begin{tabular}{|llll|}
\hline & pre-op & post-op & p value \\
\hline vas of Leg Pain & $5.90 \pm 0.98$ & $0.83 \pm 1.47$ & 0.00 \\
\hline vas of Lower Back Pain & $7.10 \pm 1.65$ & $1.21 \pm 1.99$ & 0.00 \\
\hline odi & $62.62 \pm 11.29$ & $15.17 \pm 22.05$ & 0.00 \\
\hline Intervertebral Space Height $(\mathrm{mm})$ & $10.27 \pm 1.97$ & $9.92 \pm 1.89$ & 0.04 \\
\hline Intervertebral Space Angle $\left(^{\circ}\right)$ & $6.40 \pm 3.08$ & $7.64 \pm 3.25$ & 0.01 \\
\hline Lumbar Lordosis $\left(^{\circ}\right)$ & $34.16 \pm 10.50$ & $34.34 \pm 10.75$ & 0.88 \\
\hline Foraminal Area of Operating Side $\left(\mathrm{mm}^{2}\right)$ & $132.74 \pm 38.59$ & $151.50 \pm 46.81$ & 0.04 \\
\hline Foraminal Area of Contralateral Side $\left(\mathrm{mm}^{2}\right)$ & $132.96 \pm 34.27$ & $123.19 \pm 31.92$ & 0.02 \\
\hline
\end{tabular}

Table. 4 Comparison of clinical and radiologic outcomes of L5-S1 groups pre-operative and postoperative

\begin{tabular}{|llll|}
\hline & pre-op & post-op & p value \\
\hline vas of Leg Pain & $5.91 \pm 1.03$ & $0.38 \pm 0.60$ & 0.00 \\
\hline vas of Lower Back Pain & $7.18 \pm 1.93$ & $0.59 \pm 0.70$ & 0.00 \\
\hline odi & $64.12 \pm 11.94$ & $6.12 \pm 9.37$ & 0.00 \\
\hline Intervertebral Space Height $(\mathrm{mm})$ & $10.70 \pm 1.75$ & $10.01 \pm 1.85$ & 0.00 \\
\hline Intervertebral Space Angle $\left(^{\circ}\right)$ & $10.58 \pm 5.20$ & $12.37 \pm 4.98$ & 0.02 \\
\hline Lumbar Lordosis $\left(^{\circ}\right)$ & $30.32 \pm 11.05$ & $32.30 \pm 9.46$ & 0.07 \\
\hline Foraminal Area of Operating Side $\left(\mathrm{mm}^{2}\right)$ & $130.84 \pm 30.12$ & $142.77 \pm 37.92$ & 0.03 \\
\hline Foraminal Area of Contralateral Side $\left(\mathrm{mm}^{2}\right)$ & $136.94 \pm 37.05$ & $125.85 \pm 33.15$ & 0.03 \\
\hline
\end{tabular}

\section{Figures}




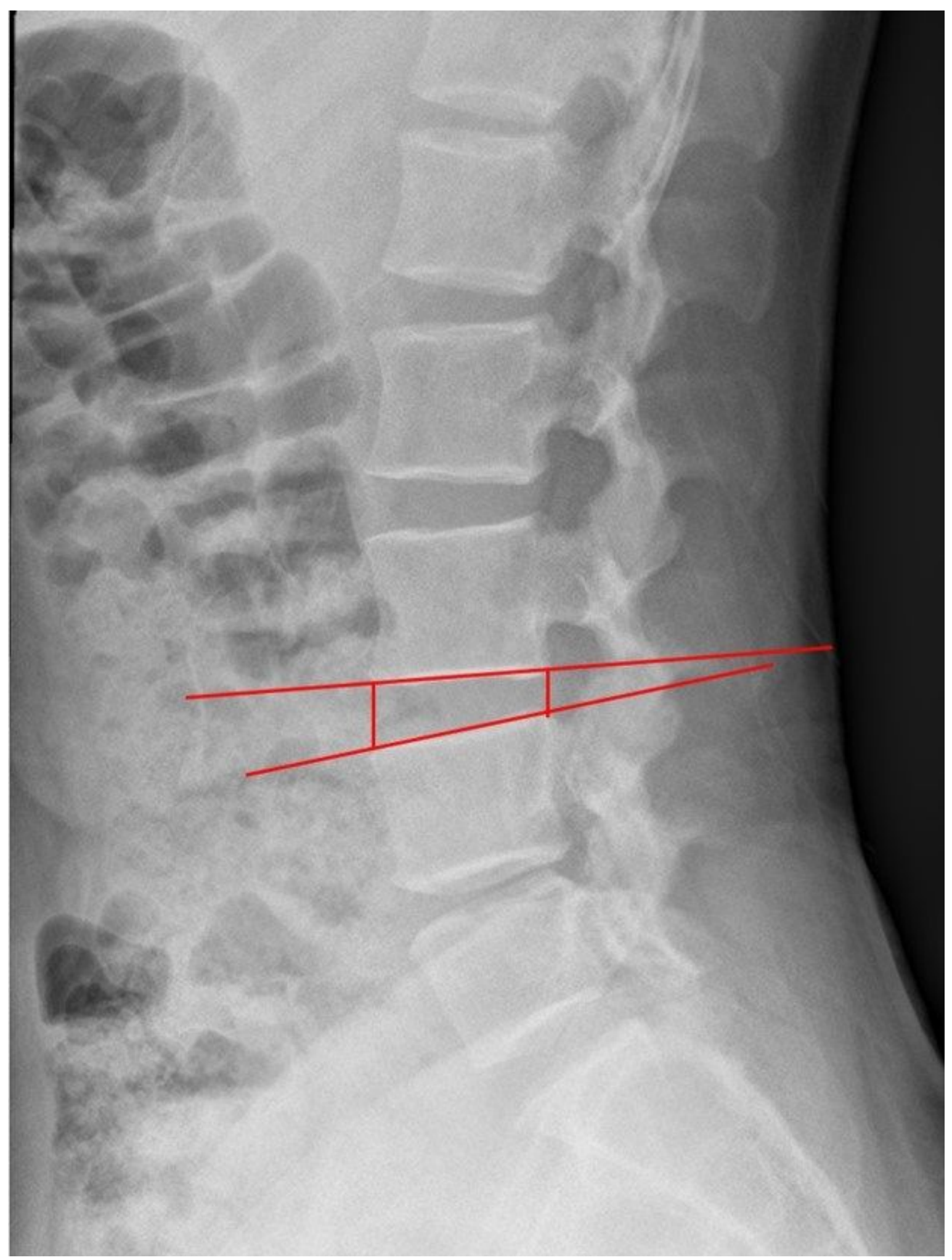

\section{Figure 1}

Intervertebral space height is the height between inferior endplate of cranial vertebra to superior end plate of caudal vertebra. Both anterior and posterior heights are measured to calculate average height. 


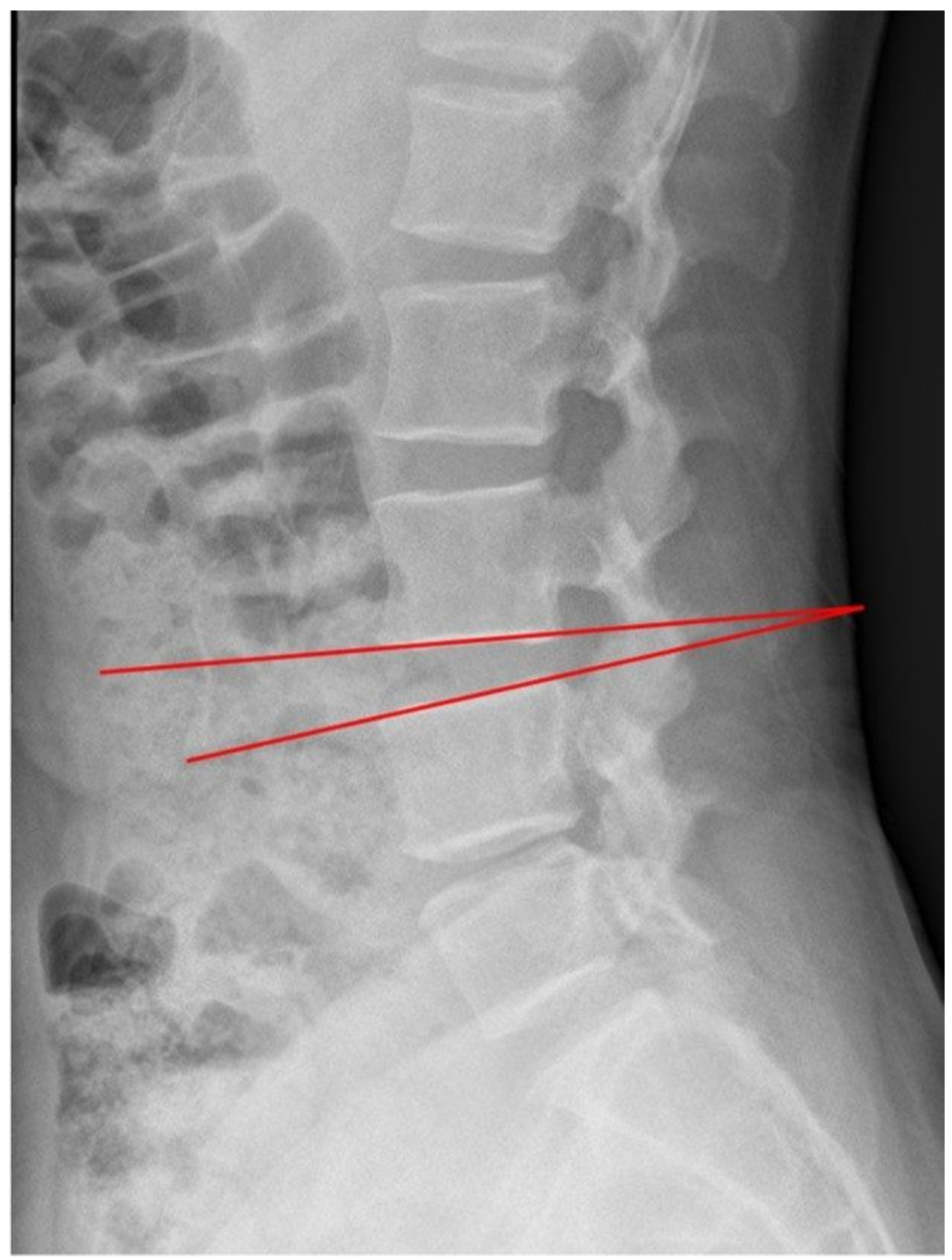

Figure 2

Intervertebral space angle is the angle of the intervertebral space measured on the X-ray film 


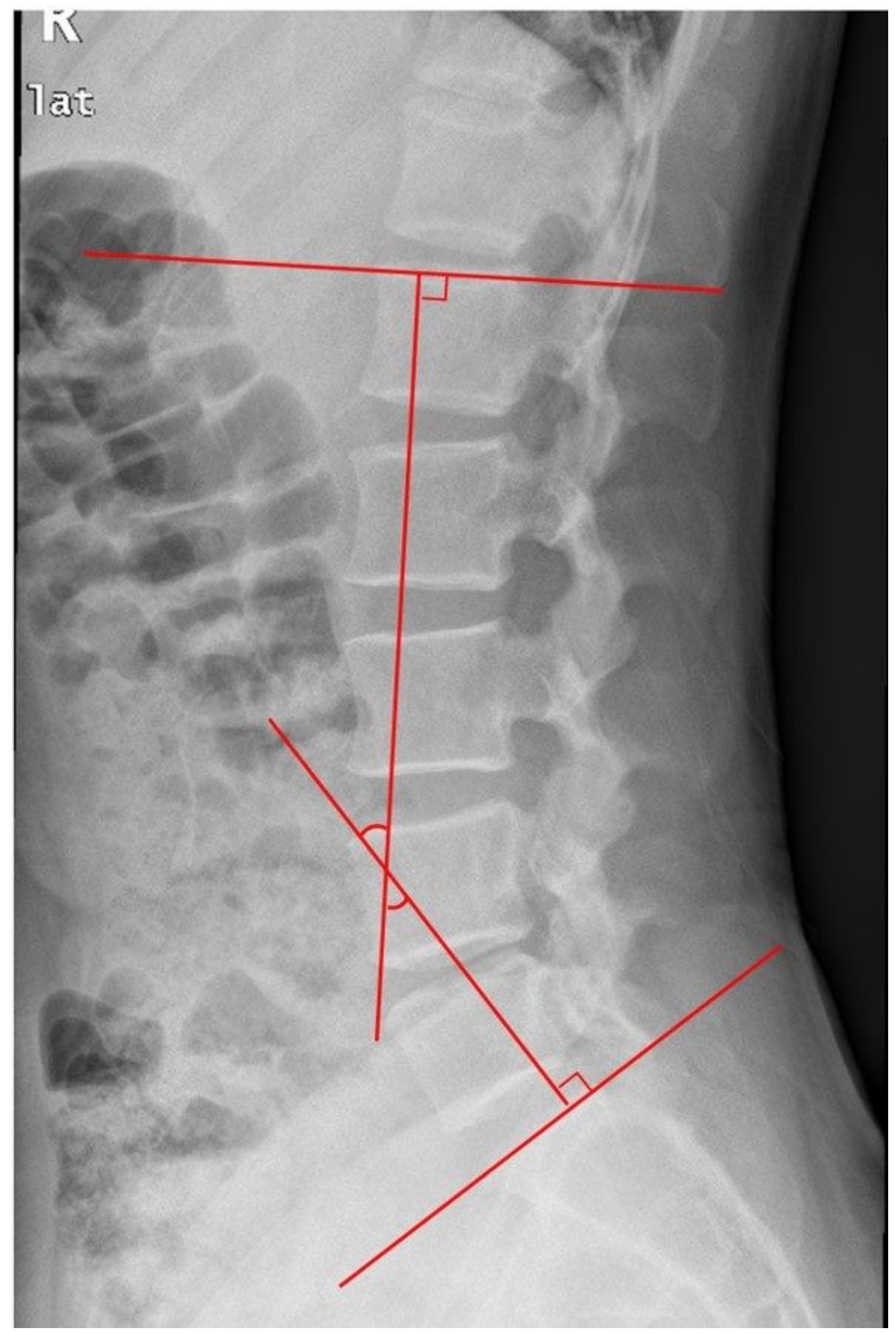

\section{Figure 3}

The lumbar lordosis angle. The lumbar lordosis angle is the angle between the vertical line of the upper edge of $\mathrm{L} 1$ and the vertical line of the upper endplate of $\mathrm{S} 1$. 


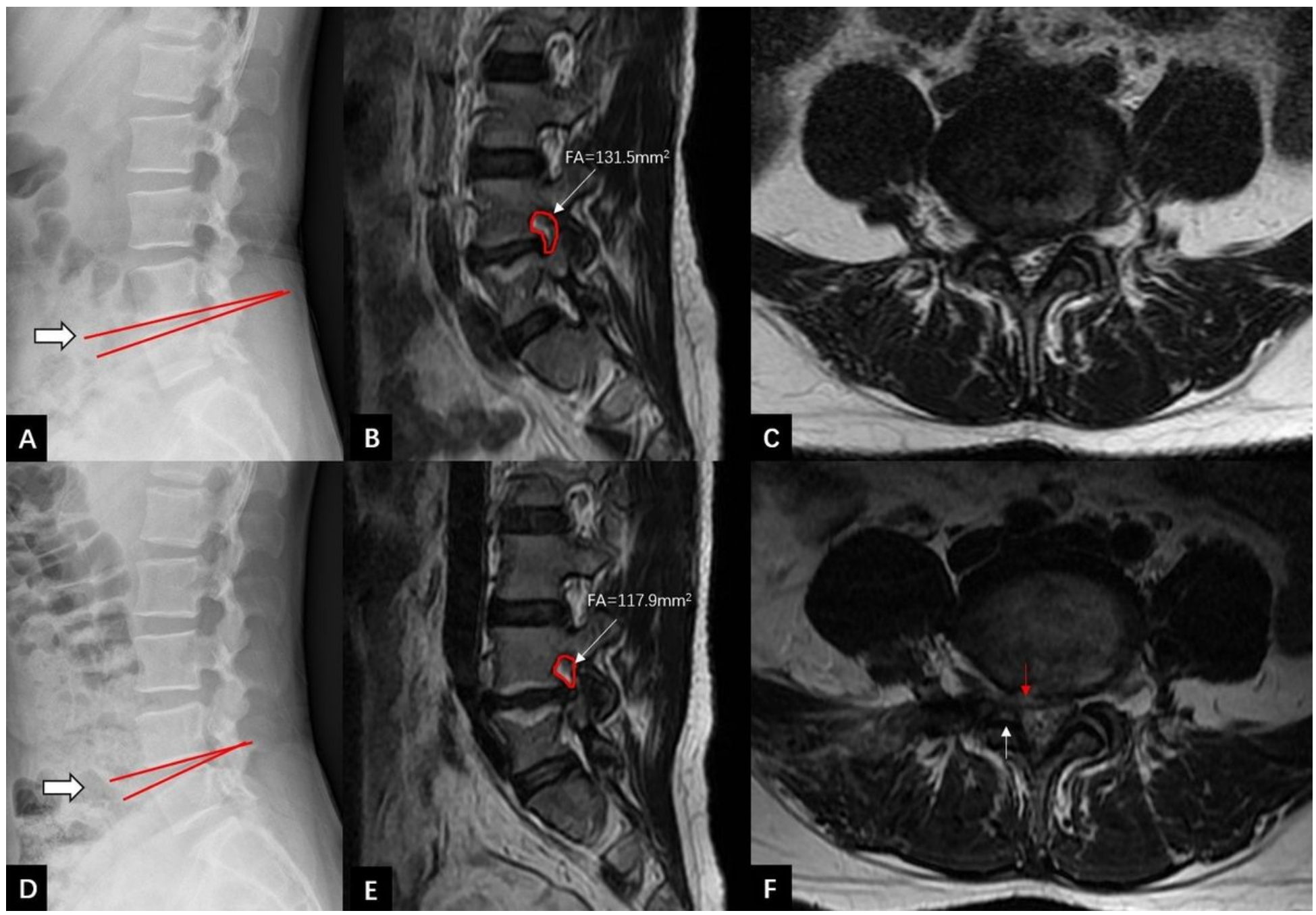

Figure 4

(A-C) Illustrated case: A 55-year-old female patient who underwent PETDF for L4/L5 disc herniation. (D) X-ray film at one-year follow-up after surgery. ISH was reduced and ISA was increased. (E) FA of the opposite side was decreased after PETDF at one-year follow-up. (F) MRI at one-week after surgery. The protruding nucleus pulposus was removed (Red arrow). The facet joint injured during foraminoplasty (White arrow). 